\title{
Détermination de la production d'acide carbonique par les ferments lactiques
}

\author{
par \\ G. WAES \\ Station Laitière de l'Etat, Melle (Belgique)
}

\section{INTRODUCTION}

La Station Laitière de l'Etat emploie depuis plusieurs années une méthode Warburg pour déterminer le pouvoir de production d'acide carbonique des ferments lactiques (Waes, 1971). Dans le cadre d'un groupe de travail FIL-IDF, Stadhouders (1975) a proposé d'apporter un certain nombre de modifications à cette méthode. Il $\mathrm{y}$ avait donc lieu d'étudier les deux méthodes de plus près.

Nous avons vérifié si la détermination de la rapidité de production d'acide carbonique d'un ferment lactique, par une méthode de laboratoire, procurait des informations sur le comportement de ce ferment dans le fromage. De plus, les deux méthodes ont fait l'objet de recherches en vue de permettre de faire un choix.

\section{MATERIEL ET METHODES}

\subsection{Lait}

La plupart des déterminations ont été faites sur du lait pasteurisé. Du lait reconstitué Nilac 10 p. 100 (Nizo, Pays-Bas) a été employé dans quelques essais.

\subsection{Ferments}

Les ferments utilisés pour les essais étaient des ferments BD, $\mathrm{B}$ et $\mathrm{D}$ provenant de maisons spécialisées. 


\subsection{Détermination de la production d'acide carbonique des ferments}

Les modifications de la méthode A proposées par Stadhouders (1975) se ramenaient à ceci :

a) Emploi de $2 \mathrm{ml}$ de lait, au lieu de $1 \mathrm{ml}$ dans les flacons Warburg.

b) Réduction du temps d'incubation de $30 \mathrm{~h}$ à $7 \mathrm{~h}$.

c) Dans la méthode $\mathrm{A}$, de l'azote pur de tout oxygène est insufflé pendant $30 \mathrm{mn}$ dans le flacon contenant le lait déjà ensemencé (de 10 p. 100 de ferment). La production d'acide carbonique peut donc commencer pendant l'insufflation de $\mathrm{N}_{2}$. L'acide carbonique dissous dans le ferment est toutefois expulsé pendant cette période.

Dans la méthode $\mathrm{B}$, nous avons utilisé des flacons à deux bras latéraux, dont l'un recevait le ferment et l'autre une solution de $\mathrm{NaHCO}_{3}$. La solution de $\mathrm{NaHCO}_{3}$ et le ferment ont été ajoutés au lait après $30 \mathrm{mn}$ d'insufflation de $\mathrm{N}_{2}$.

La méthode B peut être décrite comme suit :

La détermination a été effectuée, tout comme dans la méthode $\mathrm{A}$, à $25^{\circ} \mathrm{C}$ dans un appareil Warburg. $0,2 \mathrm{ml}$ du ferment à étudier ont été introduits dans un des bras latéraux d'un flacon Warburg spécial, l'autre bras recevant $0,2 \mathrm{ml}$ d'une solution $0,01 \mathrm{M}$ de $\mathrm{NaHCO}_{3}$. $2 \mathrm{ml}$ de lait à basse pasteurisation ont été pipettés dans le flacon. Celui-ci a été fixé au manomètre et du $\mathrm{N}_{2}$ pur de tout oxygène y a été insufflé pendant $30 \mathrm{mn}$. Le liquide contenu dans les deux bras du manomètre a été placé aussi haut que possible, de sorte que la détermination pouvait commencer sous une légère sous-pression, ce qui permettait de mesurer de plus grandes quantités de gaz. Après fermeture des flacons, le ferment et la solution de $\mathrm{NaHCO}_{3}$ étaient mélangés au lait et on procédait à la lecture. Celle-ci avait lieu toutes les heures et fut terminée après $7 \mathrm{~h}$. La détermination minimale était faite en double. Deux thermobaromètres ont été employés afin de tenir compte de la variation de température et de pression. Les calculs ont été effectués de la manière décrite par Waes (1971).

\subsection{Détermination de l'acide citrique}

L'acide citrique a été déterminé dans le fromage $(10 \mathrm{~g})$ par la méthode FIL-IDF (1971) et dans le ferment lactique ou le lait par la méthode de Galesloot et Hassing (1961). 


\subsection{Détermination des nombres de bactéries productrices d'arôme des ferments lactiques}

Les nombres totaux de bactéries productrices d'arôme ont été déterminés par la méthode modifiée de Nickels et Leesment. Le WAC $0,5 \mathrm{p} .100\left(4 \mathrm{j}\right.$ à $\left.35^{\circ} \mathrm{C}\right)$ a été employé pour $S$. diacetilactis et le WACCA 1 p. $100+0,3$ UI de pénicilline par $\mathrm{ml}\left(7 \mathrm{j}\right.$ à $\left.25^{\circ} \mathrm{C}\right)$ pour les bétacoques. De plus amples détails ont été donnés dans une publication précédente (Waes, 1968).

\section{RESULTATS}

3.1. Valeur des deux méthodes pour procurer une idée de la rapidité de production d'acide carbonique par le ferment dans le fromage

3.1.1. Provenance de l'acide carbonique mesuré au cours de la détermination

La texture du fromage Gouda est due, entre autres, au fait que les bactéries productrices d'arôme transforment, en conditions anaérobies l'acide citrique en gaz carbonique. Si la concentration de gaz dissous devient trop élevée par endroits, des yeux se forment en ces points.

Nous avons recherché si tout l'acide carbonique produit pendant l'exécution de la méthode A provenait de l'acide citrique. Ces essais ont été effectués avec deux ferments BD et deux ferments B.

Une détermination quantitative de l'acide citrique a été effectuée sur du lait de fromagerie ensemencé de 10 p. 100 de ferment lactique. Le lait ensemencé a été utilisé pour dix déterminations manométriques de la production d'acide carbonique. Le lait coagulé de dix flacons a été rassemblé après la dernière lecture (après 30 h) et l'acide citrique restant a été déterminé sur ce lait.

Compte tenu du fait que 1 mol d'acide citrique donne 2 mols de $\mathrm{CO}_{2}$, on peut calculer le nombre maximal de $\mu \mathrm{l}$ de $\mathrm{CO}_{2}$ pouvant être formés par l'acide citrique présent.

Le tableau 1 indique, pour chaque ferment, la production d'acide carbonique déterminée en 30 h par la méthode $\mathrm{A}$, le nombre calculé de $\mu \mathrm{l}$ de $\mathrm{CO}_{2}$ pouvant théoriquement provenir de la quantité d'acide citrique présente et le nombre de $\mu \mathrm{g} / \mathrm{ml}$ d'acide citrique restant dans le lait coagulé après la lecture effectuée au bout de $30 \mathrm{~h}$.

Les quantités calculées et les quantités déterminées d'acide carbonique n'ont différé que peu. La teneur en acide citrique du lait 


\section{TABLEAU 1}

Production d'acide carbonique par des ferments lactiques

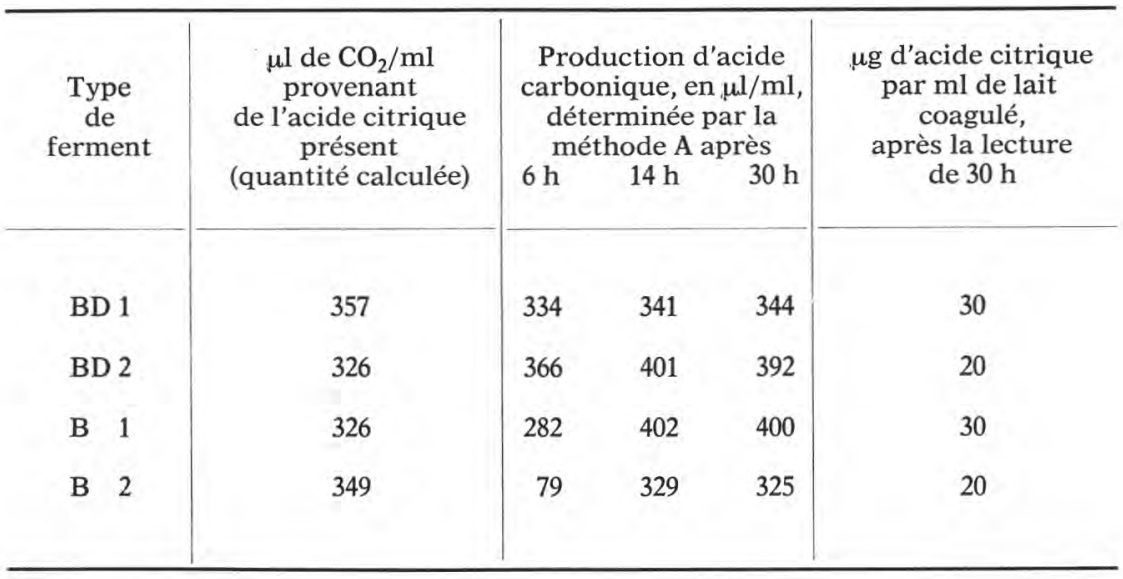

coagulé déterminée après la dernière lecture pouvait être considérée comme insignifiante. Galesloot (1961) a trouvé, par la même méthode utilisée par nous, $20 \mu \mathrm{g} / \mathrm{ml}$ d'acide citrique dans des ferments conservés depuis des jours et qui ne contenaient certainement plus d'acide citrique. On peut poser sans plus que la plus grande partie de l'acide carbonique formé au cours de la détermination provenait de l'acide citrique.

3.1.2. Relation entre la rapidité de production d'acide carbonique par un ferment lactique et la dégradation de l'acide citrique dans le fromage

La détermination de la rapidité de production d'acide carbonique d'un ferment lactique n'a d'utilité que si elle nous apprend comment cette production se déroulera dans le fromage. La détermination directe de l'acide carbonique présent dans le fromage n'est pas facile. Cet acide peut d'ailleurs provenir aussi d'autres sources que l'acide citrique. En mesurant la rapidité avec laquelle l'acide citrique se dégrade dans le fromage nous avons pu nous former une idée de la production d'acide carbonique imputable au ferment lactique.

Ces essais ont été effectués avec trois ferments BD, un ferment D et quatre ferments $\mathrm{B}$. La rapidité de production d'acide carbonique a été déterminée sur le lait de fromagerie utilisé, le jour de la fabrication du Gouda et par la méthode A. Ce Gouda a été fabriqué à la fromagerie expérimentale et par la méthode usuelle. Les déter- 


\section{TABLEAU 2}

Relation entre la production d'acide carbonique d'après la méthode $\mathrm{A}$, la dégradation de l'acide citrique et la texture du fromage Gouda

\begin{tabular}{|c|c|c|c|c|c|c|c|c|c|c|c|c|c|c|c|}
\hline \multirow{2}{*}{ Ferment } & \multicolumn{3}{|c|}{$\begin{array}{c}\text { Production de } \mathrm{CO}_{2} \\
\text { en } \mu \mathrm{l} / \mathrm{ml} \\
\text { (méthode } \mathrm{A} \text { ) }\end{array}$} & \multicolumn{11}{|c|}{ Teneur en acide citrique du fromage, en $\mu \mathrm{g} / 10 \mathrm{~g}$} & \multirow{2}{*}{$\begin{array}{c}\text { Nette } \\
\text { formation } \\
\text { d'yeux }\end{array}$} \\
\hline & $6 \mathrm{~h}$ & $13 \mathrm{~h}$ & $24 \mathrm{~h}$ & $\begin{array}{l}\text { après } \\
\text { mou- } \\
\text { lage }\end{array}$ & $\begin{array}{l}\text { après } \\
\text { pres- } \\
\text { sage }\end{array}$ & $\begin{array}{l}\text { après } \\
\text { res- } \\
\text { suyage }\end{array}$ & $\begin{array}{c}\text { après } \\
3 \mathrm{j}\end{array}$ & $\begin{array}{c}\text { après } \\
6 \mathrm{j}\end{array}$ & $\begin{array}{c}\text { après } \\
13 \mathrm{j}\end{array}$ & $\begin{array}{l}\text { après } \\
17 \mathrm{j}\end{array}$ & $\begin{array}{c}\text { après } \\
20 \mathrm{j}\end{array}$ & $\begin{array}{c}\text { après } \\
24 \mathrm{j}\end{array}$ & $\begin{array}{c}\text { après } \\
30 \mathrm{j}\end{array}$ & $\begin{array}{c}\text { après } \\
42 \mathrm{j}\end{array}$ & \\
\hline BD 1 & 280 & 340 & 341 & 15000 & 12000 & 4000 & 2000 & 0 & & & & & & & $1^{\mathrm{e}}$ semaine \\
\hline BD 2 & 314,6 & - & 323,4 & 9100 & - & 2000 & 940 & 1280 & 650 & 0 & & & & & $1^{\mathrm{e}}$ semaine \\
\hline $\mathrm{D}$ & 321,8 & 339,3 & 339,3 & 15200 & 2000 & 200 & & & & & & & & & $1^{\mathrm{e}}$ semaine \\
\hline B 1 & 50 & 282 & 353,4 & 15000 & 19600 & 15000 & 13000 & 7200 & 9200 & 8000 & 9200 & 6400 & & & $6^{\mathrm{e}}$ semaine \\
\hline B 2 & 10 & 56 & 318 & 14000 & 19600 & 13400 & 11400 & 11200 & 8000 & 6000 & 9200 & 7200 & & & $6^{e}$ semaine \\
\hline B 3 & 33 & - & 363 & 14000 & - & 10400 & 9600 & 9100 & 9550 & 8500 & 9850 & 9600 & 9850 & 9600 & $5^{\mathrm{e}}$ semaine \\
\hline B 4 & 46,3 & - & 336 & 17700 & - & 15200 & 13200 & 13800 & 9200 & 9600 & 9350 & 9300 & 9600 & 9300 & $5^{\mathrm{e}}$ semaine \\
\hline
\end{tabular}


minations de l'acide citrique restant ont été faites sur le caillé au moment du moulage et sur le fromage après le pressage, après ressuyage et au bout de $3,6,13,17,20,24,30$ et $42 \mathrm{j}$. Une photo de la coupe a été faite à chaque examen du fromage. Les résultats sont résumés dans le tableau 2.

Une très bonne corrélation a été relevée entre la rapidité de production d'acide carbonique déterminée par la méthode $\mathrm{A}$, la dégradation de l'acide citrique et la texture du fromage. Un ferment qui produisait rapidement du gaz carbonique $\left(>250 \mu \mathrm{l} \mathrm{CO}_{2}\right.$ au bout de $6 \mathrm{~h}$ ) a déterminé déjà au bout de 1 semaine un début évident de formation d'yeux dans le fromage. L'acide citrique avait disparu du fromage en l'espace de $17 \mathrm{j}$. Un ferment qui produisait lentement de l'acide carbonique $\left(<50 \mu \mathrm{l} \mathrm{CO}_{2}\right.$ au bout de $\left.6 \mathrm{~h}\right)$ n'a déterminé un début de formation d'yeux qu'au cours de la $5^{\mathrm{e}}$ semaine suivant la fabrication et la présence d'acide citrique était encore évidente dans ce fromage au bout de $42 \mathrm{j}$.

\subsubsection{Conclusion}

Il a pu être démontré par la méthode A que la plus grande partie de l'acide carbonique produit provenait de l'acide citrique. Il est apparu aussi qu'il existait une bonne corrélation entre la rapidité de production d'acide carbonique au moment de la détermination et la dégradation de l'acide citrique dans le fromage. Puisque la méthode B ne s'écartait que dans quelques détails de la méthode A, ce qui précède vaut aussi pour la méthode $B$. Les deux méthodes semblent donc convenir pour procurer une idée de la production d'acide carbonique par les ferments lactiques dans le fromage.

\subsection{Recherches sur les méthodes A et $B$}

Les deux méthodes ont fait l'objet de recherches supplémentaires. Nous avons tâché d'élaborer un mode opératoire optimal pour la méthode $\mathrm{A}$ et en ce qui concerne la méthode $\mathrm{B}$, à nous procurer une idée de l'influence exercée par l'addition de $\mathrm{NaHCO}_{3}$. Enfin, nous avons procédé à une comparaison des deux méthodes.

\subsubsection{Méthode A}

\subsubsection{Influence de la quantité de lait}

Pour la détermination il n'est employé que $1 \mathrm{ml}$ d'un mélange de lait de fromagerie et de 10 p. 100 de ferment lactique. Nous avons examiné si la reproductibilité à la lecture au bout de $7 \mathrm{~h}$ pouvait être améliorée si on portait de 1 à $2 \mathrm{ml}$ la quantité de lait ensemencé. Cet examen a été fait au moyen d'un ferment BD et de trois ferments B. La production d'acide carbonique a presque toujours été déterminée d'après cinq épreuves manométriques par essai et par ferment. Les résultats sont repris dans le tableau 3 . 


\section{TABLEAU 3}

Influence de la quantité de lait sur la reproductibilité de la méthode A

\begin{tabular}{l|c|c|c|c}
\hline \multirow{2}{*}{ Ferment } & \multicolumn{4}{|c}{$\mu 1 \mathrm{CO}_{2}$ après $7 \mathrm{~h}$} \\
\cline { 2 - 4 } & \multicolumn{2}{|c|}{$1 \mathrm{ml}$ de lait ensemencé } & \multicolumn{2}{|c}{$2 \mathrm{ml}$ de lait ensemencé } \\
\cline { 2 - 4 } & moyenne & $\begin{array}{c}\text { écart } \\
\text { standard }\end{array}$ & moyenne & $\begin{array}{c}\text { écart } \\
\text { standard }\end{array}$ \\
\cline { 2 - 4 } BD & 401 & 6 & 557 & 18 \\
B 1 & 211 & 3 & 398 & 17 \\
B 2 & 243 & 3 & 468 & 6 \\
\hline
\end{tabular}

La production d'acide carbonique était naturellement plus grande avec $2 \mathrm{ml}$, mais le classement des ferments selon l'importance de la production d'acide carbonique restait le même. La reproductibilité n'était certainement pas meilleure avec $2 \mathrm{ml}$ qu'avec $1 \mathrm{ml}$.

\subsubsection{Influence de la température}

Galesloot et Hassing (1961) ont démontré que les bétacoques se développent plus lentement par $30^{\circ} \mathrm{C}$ que par $20^{\circ} \mathrm{C}$. Nous avons examiné si l'augmentation de la température de $25^{\circ} \mathrm{C}$ à $30^{\circ} \mathrm{C}$ exerçait une influence sur la production d'acide carbonique. Ces déterminations ont été effectuées sur un ferment BD et sur deux ferments B. Leurs résultats figurent dans le tableau 4.

La production d'acide carbonique était nettement plus rapide par $30^{\circ} \mathrm{C}$ que par $25^{\circ} \mathrm{C}$ chez les ferments $\mathrm{B}$ examinés, mais n'a pas été influencée chez le ferment BD. La température d'incubation de $25^{\circ} \mathrm{C}$ a été maintenue pour les essais ultérieurs : pendant la fabrication du fromage (préparation du caillé et ressuyage) la température moyenne est certainement plus proche de $25^{\circ} \mathrm{C}$ que de $30^{\circ} \mathrm{C}$.

\subsubsection{Stabilité de la production d'acide carbonique}

La rapidité de production d'acide carbonique est-elle une caractéristique stable ? Cette rapidité ne se modifie-t-elle pas en l'espace de $14 \mathrm{j}$ ? 


\section{TABLEAU 4}

Influence de la température d'incubation sur la production d'acide carbonique

\begin{tabular}{c|c|c}
\hline \multirow{2}{*}{ Ferment } & $\begin{array}{r}\text { Production moyenne d'acide carbonique } \\
\text { après } 7 \mathrm{~h} \text { en } \mu \mathrm{c} \mathrm{CO}_{2}\end{array}$ \\
\cline { 2 - 3 } & $25^{\circ} \mathrm{C}$ & $30^{\circ} \mathrm{C}$ \\
BD & 247 & 250 \\
B 3 & 154 & 267 \\
B 6 & 128 & 187 \\
\hline
\end{tabular}

TABLEAU 5

Reproductibilité et stabilité de la production d'acide carbonique d'un ferment BD

\begin{tabular}{|c|c|c|c|c|}
\hline \multirow{3}{*}{ Jours } & \multicolumn{4}{|c|}{ Production d'acide carbonique, en $\mu \mathrm{l}$, après $7 \mathrm{~h}$ à $25^{\circ} \mathrm{C}$} \\
\hline & \multicolumn{2}{|c|}{ Lait reconstitué Nilac } & \multicolumn{2}{|c|}{ Lait pasteurisé } \\
\hline & moyenne & écart standard & moyenne & écart standard \\
\hline 0 & 355 & 4 & 303 & 7 \\
\hline 4 & 358 & 5 & 306 & 6 \\
\hline 6 & 353 & 4 & 311 & 7 \\
\hline 12 & 338 & 2 & 309 & 7 \\
\hline 14 & 350 & 2 & 299 & 6 \\
\hline
\end{tabular}

Considérant que la variation quotidienne du lait de fromagerie pourrait entraîner une variation de cette rapidité, nous avons effectué des essais aussi bien avec du lait de fromagerie qu'avec du lait reconstitué à partir de poudre Nilac. 
Chacun de ces essais ayant été effectué également en cinq répétitions, nous avons pu nous former aussi une idée de la reproductibilité de la méthode. Ces essais n'ont été effectués qu'avec un ferment BD. Le tableau 5 reproduit leurs résultats.

La rapidité de production d'acide carbonique n'a guère varié en l'espace de $14 \mathrm{j}$. Ici aussi, la reproductibilité de la méthode s'est révélée très bonne.

\subsubsection{Méthode $B$}

3.2.2.1. Influence exercée sur la production d'acide carbonique par l'addition de $\mathrm{NaHCO}_{3}$.

On sait qu'un ferment lactique contient du gaz carbonique et que celui-ci exerce un effet stimulant sur la rapidité de l'acidification. Le gaz carbonique initialement présent a été chassé par l'insufflation de $\mathrm{N}_{2}$. Nous avons étudié l'influence exercée par l'addition ou la non-addition de $\mathrm{NaHCO}_{3}$ après cette insufflation. Les essais ont été effectués, dans lesquels aussi bien $0,2 \mathrm{ml}$ d'une solution $0,01 \mathrm{M}$ de $\mathrm{NaHCO}_{3}$, que $0,2 \mathrm{ml}$ d'eau distillée ont été ajoutés dans un des bras latéraux de flacon Warburg. Deux ferments BD et trois ferments B ont été employés. Le tableau 6 donne les résultats de production d'acide carbonique après $1 \mathrm{~h}, 2 \mathrm{~h}, 7 \mathrm{~h}$ et le $\mathrm{pH}$ du lait après la dernière lecture.

L'addition de $\mathrm{NaHCO}_{3}$ a résulté après les 2 premières heures en un plus grand dégagement de gaz carbonique. Après 7 h il n'y avait plus de différence, sauf pour B8. Après un temps d'incubation, le ferment non additionné de $\mathrm{NaHCO}_{3}$ a aussi dégagé du gaz carbonique qui, à son tour, a stimulé la production ultérieure. Pour le reste, on n'a pas pu poser que l'addition de $\mathrm{NaHCO}_{3}$ limiterait la variation du $\mathrm{pH}$.

3.2.2.2. Influence du $\mathrm{NaHCO}_{3}$ sur le développement de bactéries productrices d'arôme

Nous avons examiné si l'addition de $\mathrm{NaHCO}_{3}$ stimulait le développement de bactéries productrices d'arôme. Un ferment BD et deux ferments B ont été employés pour ces essais, qui comportaient aussi l'addition soit de $0,2 \mathrm{ml}$ de $0,01 \mathrm{M} \mathrm{NaHCO}_{3}$, soit de $0,2 \mathrm{ml}$ d'eau distillée. Les nombres de producteurs d'arôme ont été déterminés sur les contenus rassemblés, après $7 \mathrm{~h}$ d'incubation, de cinq flacons manométriques. Le tableau 7 indique, pour les trois ferments, les nombres de bactéries productrices d'arôme, de $S$. diacetilactis et de bétacoques après $7 \mathrm{~h}$ d'incubation dans l'appareil Warburg, respectivement avec et sans $\mathrm{NaHCO}_{3}$. L'addition et la non-addition de $\mathrm{NaHCO}_{3}$ n'ont pas influé sur les nombres totaux de bactéries productrices d'arôme ni sur les nombres par espèce de bactéries. 
TABLEAU 6

Influence de l'addition ou de la non-addition de $\mathrm{NaHCO}_{3}$ dans la méthode $\mathrm{B}$

\begin{tabular}{|c|c|c|c|c|c|c|c|c|c|}
\hline \multirow{3}{*}{ Ferment } & \multirow{3}{*}{$\begin{array}{l}\text { Durée } \\
\text { en h }\end{array}$} & \multicolumn{4}{|c|}{$\mathrm{NaHCO}_{3}$} & \multicolumn{4}{|c|}{ Sans $\mathrm{NaHCO}_{3}$} \\
\hline & & \multicolumn{2}{|c|}{$\mu \mathrm{l} \mathrm{CO}$} & \multicolumn{2}{|r|}{$\mathrm{pH}$} & \multicolumn{2}{|r|}{$\mu \mathrm{l} \mathrm{CO}$} & \multicolumn{2}{|r|}{$\mathrm{pH}$} \\
\hline & & moyenne & écart standard & moyenne & écart standard & moyenne & ézart standard & moyenne & écart standard \\
\hline BD 5 & $\begin{array}{l}1 \\
2 \\
7\end{array}$ & $\begin{array}{r}18 \\
34 \\
478\end{array}$ & $\begin{array}{r}8 \\
18 \\
30\end{array}$ & - & - & $\begin{array}{r}7 \\
15 \\
431\end{array}$ & $\begin{array}{r}5 \\
4 \\
24\end{array}$ & - & - \\
\hline BD 6 & $\begin{array}{l}1 \\
2 \\
7\end{array}$ & $\begin{array}{r}57 \\
133 \\
672\end{array}$ & $\begin{array}{r}7 \\
13 \\
3\end{array}$ & 4,54 & 0,02 & $\begin{array}{r}61 \\
131 \\
699\end{array}$ & $\begin{array}{r}6 \\
5 \\
19\end{array}$ & 4,60 & 0 \\
\hline B 7 & $\begin{array}{l}1 \\
2 \\
7\end{array}$ & $\begin{array}{r}22 \\
36 \\
435\end{array}$ & $\begin{array}{r}2 \\
2 \\
11\end{array}$ & - & - & $\begin{array}{r}7 \\
16 \\
417\end{array}$ & $\begin{array}{l}0 \\
0 \\
6\end{array}$ & - & - \\
\hline В 8 & $\begin{array}{l}1 \\
2 \\
7\end{array}$ & $\begin{array}{r}22 \\
34 \\
188\end{array}$ & $\begin{array}{r}9 \\
16 \\
34\end{array}$ & 4,50 & 0 & $\begin{array}{r}5 \\
10 \\
113\end{array}$ & $\begin{array}{l}1 \\
1 \\
7\end{array}$ & 4,52 & 0,05 \\
\hline
\end{tabular}




\section{TABLEAU 7}

Influence du $\mathrm{NaHCO}_{3}$ sur le développement de bactéries productrices d'arôme dans la méthode B

\begin{tabular}{|c|c|c|c|}
\hline \multirow{2}{*}{ Ferment } & \multirow{2}{*}{$\begin{array}{l}\text { Espèces de bactéries } \\
\text { productrices d'arôme }\end{array}$} & \multicolumn{2}{|c|}{ Nombre par ml } \\
\hline & & avec $\mathrm{NaHCO}_{3}$ & sans $\mathrm{NaHCO}_{3}$ \\
\hline $\mathrm{BD}$ & $\begin{array}{l}\text { total } \\
\text { S. diacetilactis } \\
\text { bétacoques }\end{array}$ & $\begin{array}{r}1020000000 \\
840000000 \\
65000\end{array}$ & $\begin{array}{r}920000000 \\
880000000 \\
400000\end{array}$ \\
\hline B 1 & $\begin{array}{l}\text { total } \\
S \text {. diacetilactis } \\
\text { bétacoques }\end{array}$ & $\begin{array}{r}9200000 \\
0 \\
9000000\end{array}$ & $\begin{array}{r}9200000 \\
0 \\
11000000\end{array}$ \\
\hline B 2 & $\begin{array}{l}\text { total } \\
\text { S. diacetilactis } \\
\text { bétacoques }\end{array}$ & $\begin{array}{r}4000000 \\
0 \\
4600000\end{array}$ & $\begin{array}{r}4200000 \\
0 \\
5500000\end{array}$ \\
\hline
\end{tabular}

\subsubsection{Comparaison des deux méthodes}

Les deux méthodes ont été appliquées de la façon indiquée sous 2.3. Le temps de lecture a été limité à $7 \mathrm{~h}$. Un ferment $\mathrm{BD}$ et trois ferments B ont été utilisés pour cette étude. Le tableau 8 reproduit la valeur moyenne et l'écart standard de la production d'acide carbonique, ainsi que les valeurs $\mathrm{pH}$ après $7 \mathrm{~h}$ des différents ferments.

La production d'acide carbonique a été plus élevée avec la méthode B. Ceci correspondait aux prévisions. En ce qui concerne la rapidité de production d'acide carbonique, l'ordre de classement des quatre ferments est resté le même pour les deux méthodes. Aucune différence notable n'a été relevée quant à l'écart standard de production d'acide carbonique et de $\mathrm{pH}$.

\section{COMMENTAIRES}

Les deux méthodes conviennent pour déterminer la production d'acide carbonique d'un ferment dans le fromage. Il existait un rapport entre la rapidité de cette production dans la méthode A et la dégradation de l'acide citrique dans le fromage. Puisque les deux méthodes de détermination ne diffèrent qu'en quelques détails et comme il est apparu qu'il n'y avait pas de différence en classe- 
TABLEAU 8

Comparaison des productions d'acide carbonique et des $\mathrm{pH}$ à la fin de la lecture

\begin{tabular}{|c|c|c|c|c|c|c|c|c|}
\hline \multirow{3}{*}{ Ferment } & \multicolumn{4}{|c|}{ Méthode A } & \multicolumn{4}{|c|}{ Méthode B } \\
\hline & \multicolumn{2}{|c|}{$\begin{array}{c}\mu \mathrm{Cl} \mathrm{CO}_{2} \text { après } 7 \mathrm{~h} \\
\text { d'incubation }\end{array}$} & \multicolumn{2}{|r|}{$\mathrm{pH}$} & \multicolumn{2}{|c|}{ 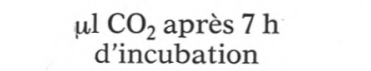 } & \multicolumn{2}{|r|}{$\mathrm{pH}$} \\
\hline & moyenne & écart standard & moyenne & écart standard & moyenne & écart standard & moyenne & écart standard \\
\hline $\mathrm{BD}$ & 316 & 2 & 4,30 & 0 & 674 & 8 & 4,45 & 0 \\
\hline B 9 & 77 & 7 & 4,45 & 0 & 118 & 11 & 4,45 & 0 \\
\hline B 10 & 182 & 15 & - & - & 406 & 45 & - & - \\
\hline B 11 & 55 & 0 & 4,36 & 0,07 & 70 & 13 & 4,42 & 0,04 \\
\hline
\end{tabular}


ment des ferments pour ce qui concerne la rapidité de production d'acide carbonique déterminée par l'une et par l'autre méthode, la méthode $\mathrm{B}$ convient certainement aussi pour cette détermination.

Il est vrai que le pouvoir de production d'acide carbonique du ferment n'est pas seul déterminant de la texture du fromage, mais il ressort, une fois de plus, de ces recherches que dans des circonstances normales ce pouvoir du ferment est le facteur principal de la formation d'yeux.

Important est aussi le fait que la production d'acide carbonique est beaucoup plus rapide dans ces deux méthodes qu'elle ne l'est dans le fromage. Dans la méthode A, par exemple, tout l'acide citrique était fermenté en l'espace de $30 \mathrm{~h}$, ce qui n'était pas le cas dans les fromages à ferments DB et B. Les fromages à ferments B contenaient même encore après $42 \mathrm{j}$ une importante quantité d'acide citrique.

Tout strictement considéré, la méthode A en apprenait suffisamment sur la rapidité de production de l'acide carbonique. La méthode B est toutefois plus standardisée et mérite donc la préférence. Quoique l'addition de $\mathrm{NaHCO}_{3}$ n'aura, dans la plupart des cas, d'effet stimulant qu'au début de la détermination, cette addition semble tout de même être une amélioration. La plupart des laboratoires ne disposent toutefois pas des indispensables flacons Warburg à deux bras latéraux.

\section{R és u m é}

L'utilité pratique de deux méthodes a été étudiée en ce qui concerne la mesure de la rapidité de production d'acide carbonique par un ferment lactique. La méthode A est en usage depuis plusieurs années dans notre laboratoire. La méthode $B$ est cette même méthode A modifiée par Stadhouders. Toutes deux sont des méthodes de Warburg, où l'on mesure dans une quantité limitée de lait (1 ou $2 \mathrm{ml}$ ) l'acide carbonique dégagé en milieu anaérobie par un ferment (culture à $10 \mathrm{p} .100$ ). Les différences entre les méthodes résidaient dans l'addition ou la non-addition d'une solution de $\mathrm{NaHCO}_{3}$ et dans l'addition du ferment avant ou après l'insufflation de $\mathrm{N}_{2}$.

Les deux méthodes conviennent pour procurer une idée du pouvoir de production d'acide carbonique des ferments lactiques employés dans la fabrication du fromage Gouda. La préférence a été accordée à la méthode $B$, plus standardisée. 


\section{S u m m a r y}

The usability of two methods permitting to measure the rate at which a starter produces carbonic acid was investigated. Method A has been used for years in our laboratory. Method B is method A modified by Stadhouders. Both are Warburg methods, in which the carbonic acid developed by a starter (10 p. 100 culture) under anaerobic circumstances is measured in a limited quantity of milk (1 or $2 \mathrm{ml}$ ). The differences between both methods consist in the addition or non-addition of a $\mathrm{NaHCO}_{3}$ solution and in the addition of the starter before or after $\mathrm{N}_{2}$ gassing. Both methods are valuable for determining the capability of starters, used in Gouda cheese, to form carbonic acid. The more standardized method B has been preferred.

Reçu pour publication en avril 1978.

\section{Bibliographie}

Galesloot (Th.) en Hassing (F.) (1961). - Enkele verschillen in gedrag tussen zuursels met als aromabacterie S. diacetilactis en L. Cremoris. Neth. Milk \& Dairy J., 15, 225.

FIL/IDF (1971). - Détermination de la teneur en acide citrique des fromages et des fromages fondus. FIL/IDF, $\mathrm{n}^{\circ} 34 \mathrm{~B}$.

StadHouders (J.) (1975). - Communication personnelle.

WAES (G.) (1968). - The enumeration of aromabacteria in BD starters. Neth. Milk \& Dairy J., 22, 29.

WAES (G.) (1971). - La production d'acide carbonique par les ferments lactiques. Le Lait, 51, 123. 